\title{
Decoupling of the DGLAP evolution equations by Laplace method
}

\author{
G.R. Boroun $*$ S. Zarrin $\dagger$ and F. Teimoury \\ Physics Department, Razi University, Kermanshah 67149, Iran
}

(Dated: July 31, 2018)

In this paper, we derive two second- order of differential equation for the gluon and singlet distribution functions by using the Laplace transform method. We decoupled the solutions of the singlet and gluon distributions into the initial conditions (function and derivative of the function) at the virtuality $Q_{0}^{2}$ separately as these solutions are defined by:

$$
\begin{aligned}
F_{2}^{s}\left(x, Q^{2}\right)= & \mathcal{F}\left(F_{s 0}, \partial F_{s 0}\right) \\
& \text { and } \\
G\left(x, Q^{2}\right)= & \mathcal{G}\left(G_{0}, \partial G_{0}\right) .
\end{aligned}
$$

We compared our results with the MSTW parameterization and the experimental measurements of $F_{2}^{p}\left(x, Q^{2}\right)$.

\section{INTRODUCTION}

The Dokshitzer-Gribov-Lipatov-Altarelli-Parisi (DGLAP) [1-3] evolution equations are a set of the integro-differential equations as can be evaluated the parton distribution functions (PDFs) into the $Q^{2}$ evolution (where $Q^{2}$ is the four momenta transfer in a deep inelastic scattering (DIS) process). The DIS structure functions provide our information about the deep structure of the hadrons and parton densities at collider and coefficient functions which contain information about the boson-parton interaction. These evolution equations are fundamental tools to test perturbative quantum chromodynamics (pQCD) in DIS experiments where the density functions can be evaluated by solving the DGLAP equations into the initial distributions.

The structure function $F_{2}\left(x, Q^{2}\right)$ reflects the momentum distributions of the partons in the nucleon. But the gluon distribution $G\left(x, Q^{2}\right)$ does not appear in the experimental directly, and it is determined only through the quark distributions in conjunction with the DGLAP evolution equations. It is also important to know the gluon distribution inside a hadron at low $x$ because gluons are expected to be dominate in this region. Traditionally, gluon and quark distribution functions have been determined using the two coupled integral-differential (DGLAP) equations. The solutions of the unpolarized DGLAP evolution equations decoupled have been discussed considerably over the last years [4-7]. There exist two main classes of approaches: those that solve the equation indirectly in $s$-space at the Laplace transform method [4-6], and those that decouple the DGLAP equations directly in x-space with define a hard pomeron behavior for the distribution functions [7]. In Refs.[4-6], authors have derived the gluon distribution and the singlet structure function into the initial conditions $G_{0}\left(x, Q_{0}^{2}\right)$ and $F_{s 0}\left(x, Q_{0}^{2}\right)$. The decoupled solutions for the gluon distribution and the singlet structure function determined by

$$
F_{2}^{s}\left(x, Q^{2}\right)=\mathcal{F}\left(F_{s 0}, G_{0}\right) \quad \text { and } \quad G\left(x, Q^{2}\right)=\mathcal{G}\left(F_{s 0}, G_{0}\right) .
$$

where the functions $\mathcal{F}$ and $\mathcal{G}$ are determined by the splitting functions into the both initial conditions. However this method is not general, because solutions of the coupled DGLAP equations

\footnotetext{
*Electronic address: boroun@razi.ac.ir; grboroun@gmail.com
}

${ }^{\dagger}$ Electronic address: salah.zarin@gmail.com 
are possible under certain conditions. In Ref.[7], a set of formulae to extract two second-order independent differential equation for the gluon and singlet distribution functions have derived. In this method, the singlet quark and gluon distributions have the same high-energy behavior at low$x$ according to the hard- pomeron behavior for parton distributions. Decoupling of the DGLAP evolution equations, for the functions $F_{2}^{s}\left(x, Q^{2}\right)$ and $G\left(x, Q^{2}\right)$, determined as a function of their initial parameterisation at the starting scale $Q_{0}^{2}$ as

$$
F_{2}^{s}\left(x, Q^{2}\right)=\mathcal{F}\left(\partial F_{s 0}, F_{s 0}\right) \quad \text { and } \quad G\left(x, Q^{2}\right)=\mathcal{G}\left(\partial G_{0}, G_{0}\right),
$$

but this method is dependence to the model behavior for the parton distribution functions.

Now we would like to find an explicit and general solution for the decoupling of the DGLAP evolution equations. This method is independent of the parton distribution behavior. In this paper, the DGLAP evolution equations coupled convert to the two second order differential equation which define $G\left(x, Q^{2}\right)$ and $F_{2}^{s}\left(x, Q^{2}\right)$ in terms of the individual initial conditions $\left\{G\left(x, Q_{0}^{2}\right), \partial G\left(x, Q_{0}^{2}\right)\right\}$ and $\left\{F_{2}^{s}\left(x, Q_{0}^{2}\right), \partial F_{2}^{s}\left(x, Q_{0}^{2}\right)\right\}$ respectively.

\section{METHOD}

The DGLAP evolution equations can be written by

$$
\begin{gathered}
\frac{4 \pi}{\alpha_{s}\left(Q^{2}\right)} \frac{\partial F_{2}^{s}\left(x, Q^{2}\right)}{\partial \ln \left(Q^{2}\right)}=\left[4+\frac{16}{3} \ln \left(\frac{1-x}{x}\right)\right] F_{2}^{s}\left(x, Q^{2}\right)+\frac{16 x}{3} \int_{x}^{1}\left(\frac{F_{2}^{s}\left(z, Q^{2}\right)}{z}-\frac{F_{2}^{s}\left(x, Q^{2}\right)}{x}\right) \frac{d z}{z-x} \\
-\frac{8}{3} x \int_{x}^{1} F_{2}^{s}\left(z, Q^{2}\right)\left(1+\frac{x}{z}\right) \frac{d z}{z^{2}}+2 n_{f} x \int_{x}^{1} G\left(z, Q^{2}\right)\left(1-2 \frac{x}{z}+2 \frac{x^{2}}{z^{2}}\right) \frac{d z}{z^{2}},
\end{gathered}
$$

and

$$
\begin{gathered}
\frac{4 \pi}{\alpha_{s}\left(Q^{2}\right)} \frac{\partial G\left(x, Q^{2}\right)}{\partial \ln \left(Q^{2}\right)}=\left[\frac{33-2 n_{f}}{3}+12 \ln \left(\frac{1-x}{x}\right)\right] G\left(x, Q^{2}\right)+12 x \int_{x}^{1}\left(\frac{G\left(z, Q^{2}\right)}{z}-\frac{G\left(x, Q^{2}\right)}{x}\right) \frac{d z}{z-x} \\
+12 x \int_{x}^{1} G\left(z, Q^{2}\right)\left(\frac{z}{x}-2+\frac{x}{z}-\frac{x^{2}}{z^{2}}\right) \frac{d z}{z^{2}}+\frac{8}{3} \int_{x}^{1} F_{2}^{s}\left(z, Q^{2}\right)\left(1+\left(1-\frac{x}{z}\right)^{2}\right) \frac{d z}{z} .
\end{gathered}
$$

These equations are coupled into the singlet and gluon structure functions. Let us introduce the variables $v \equiv \ln (1 / x)$ and $w \equiv \ln (1 / z)$ and note that $F_{2}^{s}\left(e^{-v}, Q^{2}\right) \equiv \hat{F}_{2}^{s}\left(v, Q^{2}\right)$ and $G\left(e^{-v}, Q^{2}\right) \equiv$ $\hat{G}\left(v, Q^{2}\right)$. Using Laplace transform method in $v$-space [6-6,8-10], we can define the distribution functions in $s$-space as

$f\left(s, Q^{2}\right)=\mathcal{L}\left[\hat{F}_{2}^{s}\left(v, Q^{2}\right) ; s\right]=\int_{0}^{\infty} \hat{F}_{2}^{s}\left(v, Q^{2}\right) e^{-s v} d v, g\left(s, Q^{2}\right)=\mathcal{L}\left[\hat{G}\left(v, Q^{2}\right) ; s\right]=\int_{0}^{\infty} \hat{G}_{s}\left(v, Q^{2}\right) e^{-s v} d v$,

and

$$
\mathcal{L}\left[\frac{\partial \hat{F}_{2}^{s}}{\partial w}\left(w, Q^{2}\right) ;\right]=s f\left(s, Q^{2}\right), \quad \mathcal{L}\left[\frac{\partial \hat{G}_{s}}{\partial w}\left(w, Q^{2}\right) ;\right]=s g\left(s, Q^{2}\right)
$$


We know that $F_{2}^{s}\left(v=0, Q^{2}\right)=G\left(v=0, Q^{2}\right)=0$ and introduce the new variable $\tau$ as $\frac{d \tau\left(Q^{2}\right)}{d \log \left(Q^{2}\right)}=$ $\frac{\alpha_{s}\left(Q^{2}\right)}{4 \pi}$ for the $Q^{2}$ dependence. Therefore, the coupled first order differential equations in $s$-space can be rewritten:

$$
\frac{\partial f}{\partial \tau}(s, \tau)=\Phi_{f}(s) f(s, \tau)+\Theta_{f}(s) g(s, \tau)
$$

and

$$
\frac{\partial g}{\partial \tau}(s, \tau)=\Phi_{g}(s) g(s, \tau)+\Theta_{g}(s) f(s, \tau)
$$

where the coefficient functions $\Theta(s)$ and $\Phi(s)$ are [4]

$$
\begin{gathered}
\Phi_{f}(s)=4-\frac{8}{3}\left(\frac{1}{s+1}+\frac{1}{s+2}+2\left(\psi(s+1)+\gamma_{E}\right)\right), \\
\Theta_{f}(s)=2 n_{f}\left(\frac{1}{s+1}-\frac{2}{s+2}+\frac{2}{s+3}\right), \\
\Phi_{g}(s)=\frac{33-2 n_{f}}{3}+12\left(\frac{1}{s}-\frac{2}{s+1}+\frac{1}{s+2}-\frac{1}{s+3}-\psi(s+1)-\gamma_{E}\right),
\end{gathered}
$$

and

$$
\Theta_{g}(s)=\frac{8}{3}\left(\frac{2}{s}-\frac{2}{s+1}+\frac{1}{s+2}\right) .
$$

Here $\psi(s)$ is the digamma function, $\gamma_{E}=0.5772156 \ldots$ is Euler's constant and $n_{f}$ is the number of quark flavors. Now let us find our solution for decoupling of the functions $f$ and $g$ from the above equations. The explicit form of the two second order differential equation in $s$-space are given by

$$
\frac{\partial^{2} \digamma}{\partial^{2} \tau}(s, \tau)-\left(\Phi_{f}(s)+\Phi_{g}(s)\right) \frac{\partial \digamma}{\partial \tau}(s, \tau)-\left(\Theta_{f}(s) \Theta_{g}(s)-\Phi_{f}(s) \Phi_{g}(s)\right) \digamma(s, \tau)=0, \quad(\digamma=f \text { or } g) .
$$

Solving these equations, we obtain two independent solution as

$$
f(s, \tau)=c_{1} \exp \left(\frac{1}{2}\left(R_{1}(s)+R(s)\right) \tau\right)+c_{2} \exp \left(\frac{1}{2}\left(R_{1}(s)-R(s)\right) \tau\right),
$$

and

$$
g(s, \tau)=c_{1}^{\prime} \exp \left(\frac{1}{2}\left(R_{1}(s)+R(s)\right) \tau\right)+c_{2}^{\prime} \exp \left(\frac{1}{2}\left(R_{1}(s)-R(s)\right) \tau\right)
$$

where $R_{1}(s)=\Phi_{f}(s)+\Phi_{g}(s), R(s)=\sqrt{\left(\Phi_{f}(s)-\Phi_{g}(s)\right)^{2}+4 \Theta_{g} \Theta_{f}}$ and the coefficients $c$ and $c^{\prime}$ are the initial conditions (for the function and the derivative of function) at the $Q_{0}^{2}$ value. Therefore our solutions can be find

$$
f(s, \tau)=k_{1}(s, \tau) f(s, 0)+\left.k_{2}(s, \tau) \frac{\partial f(s, \tau)}{\partial \tau}\right|_{\tau=0},
$$

and

$$
g(s, \tau)=k_{1}(s, \tau) g(s, 0)+\left.k_{2}(s, \tau) \frac{\partial g(s, \tau)}{\partial \tau}\right|_{\tau=0},
$$


where $g(s, 0) \equiv g\left(s, Q_{0}^{2}\right)$ and $f(s, 0) \equiv f\left(s, Q_{0}^{2}\right)$ and the coefficient functions are defined by

$k_{1}(s, \tau)=-\frac{1}{R(s)}\left[\left(R(s)+R_{1}(s)\right) \exp \left(\frac{1}{2} R_{1}(s) \tau\right) \sinh \left(\frac{1}{2} R(s) \tau\right)-R(s) \exp \left(\frac{1}{2}\left(R(s)+R_{1}(s)\right) \tau\right)\right]$,

and

$$
k_{2}(s, \tau)=\frac{1}{R(s)}\left[2 \exp \left(\frac{1}{2} R_{1}(s) \tau\right) \sinh \left(\frac{1}{2} R(s) \tau\right)\right]
$$

In $v$-space, we introduce two kernels $\hat{K}_{1}(v, \tau)$ and $\hat{K}_{2}(v, \tau)$ with respect to the inverse Laplace transforms of the coefficients $k_{1}(s, \tau)$ and $k_{2}(s, \tau)$, as

$$
\hat{K}_{1}(v, \tau) \equiv \mathcal{L}^{-1}\left[k_{1}(s, \tau) ; v\right] \quad, \quad \hat{K}_{2}(v, \tau) \equiv \mathcal{L}^{-1}\left[k_{2}(s, \tau) ; v\right] .
$$

Also we can consider these two parameters at the initial scale $Q^{2}=Q_{0}^{2}$ (i.e., $\tau=0$ ) where $\hat{K}_{1}(v, 0)=\delta(v)$ and $\hat{K}_{2}(v, 0)=0$. Nothing that the inverse Laplace transforms of the parton distributions at the initial scale are given by

$$
\hat{F}_{2}^{s}\left(v, Q_{0}^{2}\right)=\mathcal{L}^{-1}\left[f\left(s, Q_{0}^{2}\right)\right] \quad, \quad \hat{G}\left(v, Q_{0}^{2}\right)=\mathcal{L}^{-1}\left[g\left(s, Q_{0}^{2}\right)\right] .
$$

Finally, we can obtain our general solutions for the decoupling of the singlet and gluon distributions with respect to Eqs.14 in $x$-space in the following forms

$$
F_{2}^{s}\left(x, Q^{2}\right)=\int_{x}^{1} K_{1}\left(\frac{x}{y}, \tau\right) F_{2}^{s}\left(y, Q_{0}^{2}\right) \frac{d y}{y}+\left.\int_{x}^{1} K_{2}\left(\frac{x}{y}, \tau\right) \frac{\partial F_{2}^{s}(y, \tau)}{\partial \tau}\right|_{Q^{2}=Q_{0}^{2}} \frac{d y}{y},
$$

and

$$
G\left(x, Q^{2}\right)=\int_{x}^{1} K_{1}\left(\frac{x}{y}, \tau\right) G\left(y, Q_{0}^{2}\right) \frac{d y}{y}+\left.\int_{x}^{1} K_{2}\left(\frac{x}{y}, \tau\right) \frac{\partial G(y, \tau)}{\partial \tau}\right|_{Q^{2}=Q_{0}^{2}} \frac{d y}{y}
$$

or, in general form, we can rewrite the decoupled solutions as a reduced solution with the same coefficient functions as

$$
\mathbb{F}\left(x, Q^{2}\right)=\int_{x}^{1} K_{1}\left(\frac{x}{y}, \tau\right) \mathbb{F}\left(y, Q_{0}^{2}\right) \frac{d y}{y}+\left.\int_{x}^{1} K_{2}\left(\frac{x}{y}, \tau\right) \frac{\partial \mathbb{F}(y, \tau)}{\partial \tau}\right|_{Q^{2}=Q_{0}^{2}} \frac{d y}{y}, \quad\left(\mathbb{F}=F_{2}^{s} \text { or } G\right) .
$$

To get the solution this general equation, we need to the input initial conditions $\left(\mathbb{F}_{0}\right.$ and $\left.\partial \mathbb{F}_{0}\right)$ individual for any distribution function according to the Refs.[11-12]. These results are general and gives us the exact functions of $x$ and $Q^{2}$ in a domain $x_{\min } \leq x \leq x_{\max }$ and $Q_{\min }^{2} \leq Q^{2} \leq Q_{\max }^{2}$.

\section{RESULTS AND CONCLUSION}

In this method we have determined the $x$ space distribution functions individual with respect to the initial and derivative of initial conditions at the stating point. We have constructed a general solution for the DGLAP evolution equations for the gluon and singlet distributions into the initial conditions respectively. To determine of the proton structure function we need to know the initial singlet structure functions only and also the initial gluon distribution functions for the gluon density which are given in Appendix A. For the validity and compatibility of the two sets of analytical solutions for the singlet structure function and gluon distribution function, we compared 
the distribution functions with the H1 data [14] and with the MSTW numerical solutions [13]. We compared the solutions of Eqs.(19) and (20) for the singlet structure function and gluon distribution respectively with the MSTW distributions in Figs.1 and 2. Here the input for $\left\{F_{2}^{s}\left(x, Q_{0}^{2}\right), \partial F_{2}^{s}\left(x, Q_{0}^{2}\right)\right\}$ and $\left\{G\left(x, Q_{0}^{2}\right), \partial G\left(x, Q_{0}^{2}\right)\right\}$ are taken from the Eqs.A1-A5 corresponding to the lowest- $Q^{2}$ point. In Fig.3 we have compared our results for the proton structure function with the H1 collaboration [14] data at small $x$ at different $Q^{2}$ values. We consider the range $45 \leq Q^{2} \leq 800 \mathrm{GeV}^{2}$ and $0.0004<x<0.05$ for the experimental data. In the general solutions of singlet structure function our computed values of $F_{2}^{s}\left(x, Q^{2}\right)$ from Eq.(19) are plotted against $x$ for different values of $Q^{2}$. In these graphs, it is observed that the best-fit curves are obtained for $F_{2}^{p}\left(x, Q^{2}\right) \simeq \frac{5}{18} F_{2}^{s}\left(x, Q^{2}\right)$ as inputs for $\left\{F_{2}^{s}\left(x, Q_{0}^{2}\right), \partial F_{2}^{s}\left(x, Q_{0}^{2}\right)\right\}$ are taken from Eqs.A1-A3. It can be seen that our results are in good consistency with the H1 data.

In conclusion, we obtained two decoupling analytical evolution equation, as they are two homogeneous second-order differential equation, for the singlet $F_{2}^{s}\left(x, Q^{2}\right)$ and gluon $G\left(x, Q^{2}\right)$ distribution functions from the linear DGLAP evolution equations by using the Laplace transform method, respectively. These equations are general and require only a knowledge individual $F_{2}^{s}\left(x, Q_{0}^{2}\right)$, $G_{0}\left(x, Q_{0}^{2}\right)$ and those derivatives at the starting value $Q_{0}^{2}$ for the evolution, respectively. We observed that the general solutions are in good consistency with the experimental data and parameterizations. 


\section{References}

1. Yu. L.Dokshitzer, Sov.Phys.JETPG 6, 641(1977 ).

2. G.Altarelli and G.Parisi, Nucl.Phys.B126, 298(1997).

3. V.N.Gribov and L.N.Lipatov, Sov.J.Nucl.Phys.28, 822(1978).

4.M.M.Block, L.Durand, P.Ha D.W.Mckay, Phys.Rev.D83, 054009(2011).

5.M.M.Block, L.Durand, P.Ha and D.W.Mckay, arXiv:1004.1440(2010).

6.M.M.Block, L.Durand, P.Ha D.W.Mckay, Eur.Phys.J.C69, 425(2010).

7.G.R.Boroun and B.Rezaei,Eur. Phys. J. C73, 2412(2013).

8.M.M. Block, Eur. Phys. J. C, 65, 1(2010).

9.M.M. Block, L. Durand, and D.W. McKay, Phys. Rev. D, 79, 014031(2009).

10.M.M. Block, L. Durand, and D.W. McKay, Phys. Rev. D, 77, 094003(2008).

11.M.M. Block, E.L. Berger and C-I. Tan, Phys. Rev. Lett. 97, 252003(2006).

12. E.L. Berger, M.M. Block and C-I. Tan, Phys. Rev. Lett. 98, 242001(2007).

13.A. D. Martin, W. J. Stirling, R. S. Thorne, and G. Watt, Eur. Phys. J. C 63, 189 (2009).

14.V. Andreev and et al. [H1 Collaboration], Eur. Phys. J. C 74, 2814(2014). 


\section{Appendix A}

In Refs.[11-12] the proton structure function $F_{2}^{p}$ for $0<x<x_{P}$ has been parameterized in $x$-space as

$$
\begin{aligned}
F_{2}^{p}\left(x, Q^{2}\right)= & (1-x)\left(\frac{F_{P}}{1-x_{P}}+\left(a_{0}+a_{1} \ln \left(Q^{2}\right)+a_{2} \ln ^{2}\left(Q^{2}\right)\right) \ln \left[\frac{x_{P}(1-x)}{x\left(1-x_{P}\right)}\right]\right. \\
& \left.+\left(b_{0}+b_{1} \ln \left(Q^{2}\right)+b_{2} \ln ^{2}\left(Q^{2}\right)\right) \ln ^{2}\left[\frac{x_{P}(1-x)}{x\left(1-x_{P}\right)}\right]\right)
\end{aligned}
$$

where $x_{P}=0.09$ and $F_{2}^{p}\left(x_{P}, Q^{2}\right)=F_{P}=0.41$. Table I shows fitted quantities and their errors. In region $x<0.1$ we can approximately write $F_{2}^{s}\left(x, Q^{2}\right) \simeq 18 / 5 F_{2}^{p}\left(x, Q^{2}\right)$. So in $Q_{0}^{2}=1$ we have

$$
\left.\frac{\partial F_{2}^{p}}{\partial \tau}\left(x, Q^{2}\right)\right|_{Q^{2}=Q_{0}^{2}} \simeq \frac{4 \pi}{\alpha_{s}\left(Q_{0}^{2}\right)}(1-x)\left[a_{1} \ln \left(\frac{x_{P}(1-x)}{x\left(1-x_{P}\right)}\right)+b_{1} \ln ^{2}\left(\frac{x_{P}(1-x)}{x\left(1-x_{P}\right)}\right)\right],
$$

or

$$
\left.\frac{\partial F_{2}^{s}}{\partial \tau}\left(x, Q^{2}\right)\right|_{Q^{2}=Q_{0}^{2}} \simeq \frac{72 \pi}{5 \alpha_{s}\left(Q_{0}^{2}\right)}(1-x)\left[a_{1} \ln \left(\frac{x_{P}(1-x)}{x\left(1-x_{P}\right)}\right)+b_{1} \ln ^{2}\left(\frac{x_{P}(1-x)}{x\left(1-x_{P}\right)}\right)\right] .
$$

Also, the LO gluon distribution function $G\left(x, Q^{2}\right)=x g\left(x, Q^{2}\right)$ has been determined by

$$
\begin{gathered}
G\left(x, Q^{2}\right)=c_{0}+c_{1} \ln \left(Q^{2}\right)+c_{2} \ln ^{2}\left(Q^{2}\right)+\left[d_{0}+d_{1} \ln \left(Q^{2}\right)+d_{2} \ln ^{2}\left(Q^{2}\right)\right] \ln \left(\frac{1}{x}\right) \\
{\left[e_{0}+e_{1} \ln \left(Q^{2}\right)+e_{2} \ln ^{2}\left(Q^{2}\right)\right] \ln ^{2}\left(\frac{1}{x}\right) .}
\end{gathered}
$$

The parameters have been shown in Table I. The derivative of LO gluon distribution function in $Q_{0}^{2}=1$ becomes

$$
\left.\frac{\partial G}{\partial \tau}\left(x, Q^{2}\right)\right|_{Q^{2}=Q_{0}^{2}}=\frac{4 \pi}{\alpha_{s}\left(Q_{0}^{2}\right)}\left(c_{1}+d_{1} \ln \left(\frac{1}{x}\right)+e_{1} \ln ^{2}\left(\frac{1}{x}\right)\right) .
$$


TABLE I: The fitted parameters

\begin{tabular}{lccc}
\hline \hline & & \\
parameters & value & parameters & value \\
& & & \\
& & & \\
$c_{0}$ & -0.459 & $a_{0}$ & $-5.381 \times 10^{-2} \pm 2.17 \times 10^{-3}$ \\
$c_{1}$ & -0.143 & $a_{1}$ & $2.034 \times 10^{-2} \pm 1.19 \times 10^{-3}$ \\
$c_{2}$ & -0.0155 & $a_{2}$ & $4.999 \times 10^{-3} \pm 2.23 \times 10^{-4}$ \\
$d_{0}$ & 0.231 & & \\
$d_{1}$ & 0.00971 & $b_{0}$ & $9.955 \times 10^{-3} \pm 3.09 \times 10^{-4}$ \\
$d_{2}$ & -0.0147 & $b_{1}$ & $3.810 \times 10^{-3} \pm 1.73 \times 10^{-4}$ \\
& & & $9.923 \times 10^{-4} \pm 2.85 \times 10^{-5}$ \\
$e_{0}$ & 0.0836 & & \\
$e_{1}$ & 0.06328 & & \\
$e_{2}$ & 0.0112 & & \\
\hline
\end{tabular}




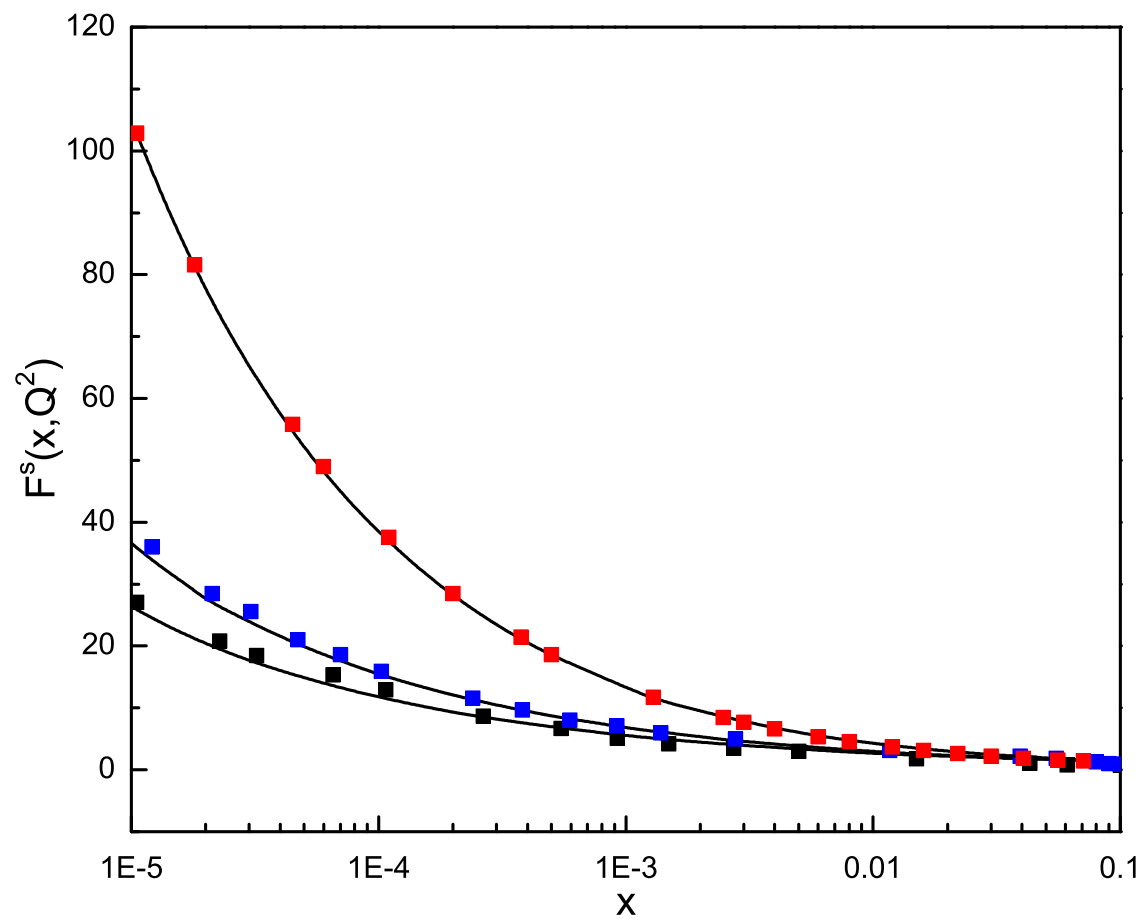

FIG. 1: The singlet structure function compared with the MSTW parameterization [13](solid line) at $Q^{2}=$ 20, 100 and $M_{z}^{2} \mathrm{GeV}^{2}$ ( bottom to top, respectively).

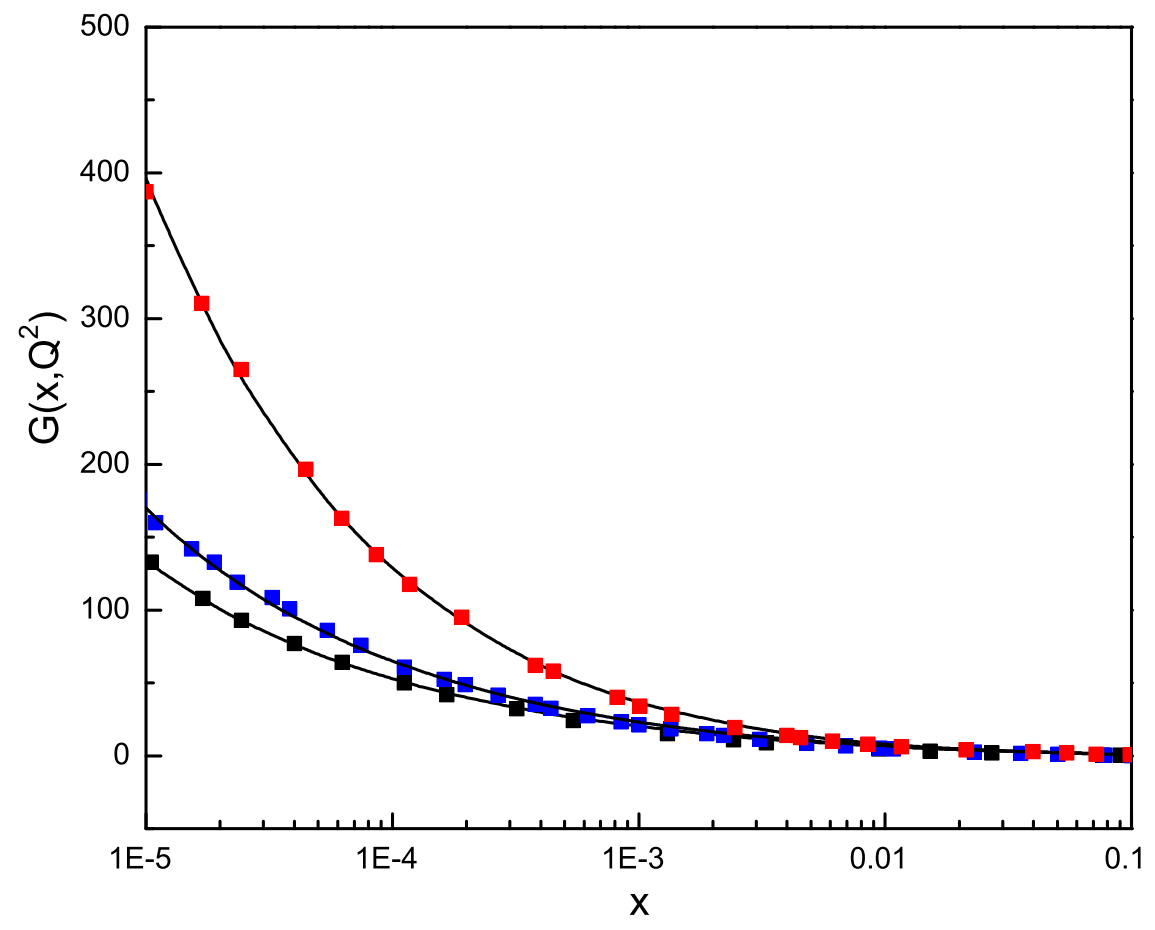




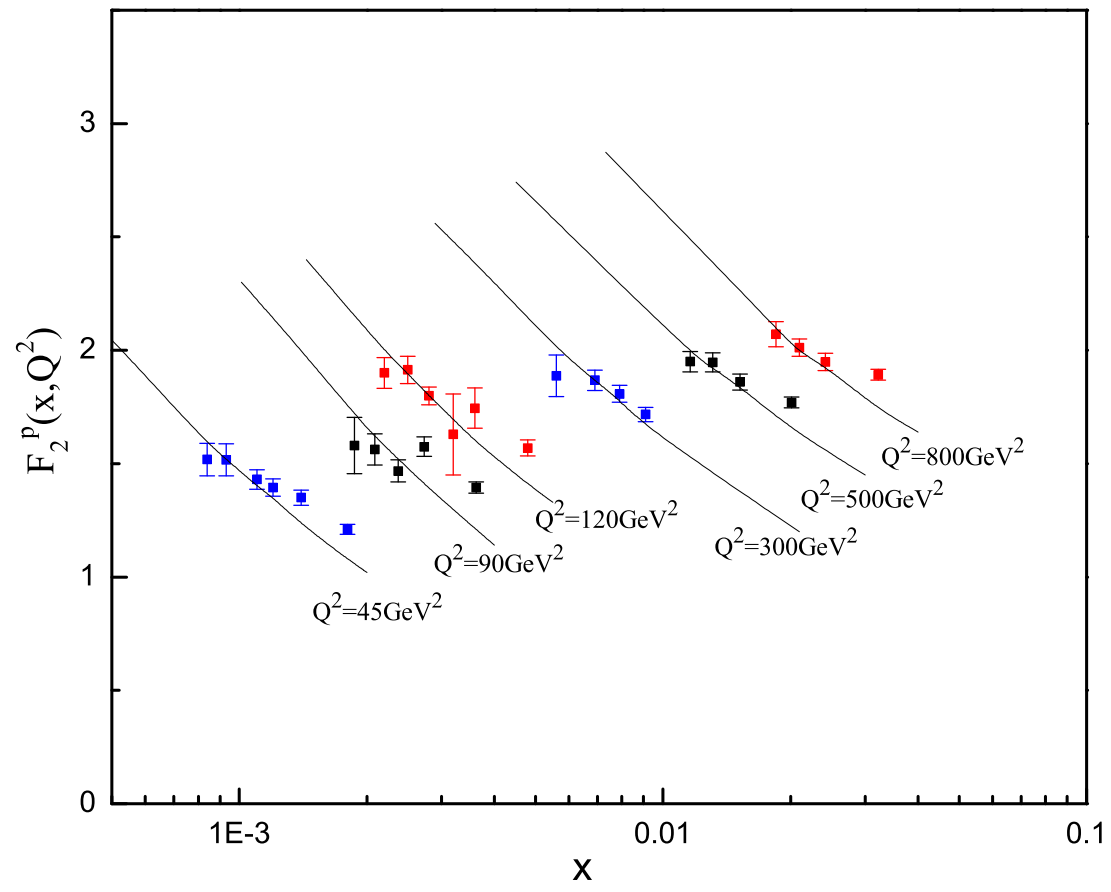

FIG. 3: Proton structure function $F_{2}^{p}\left(x, Q^{2}\right)$ as function of $x$ at different $Q^{2}$ values compared with the H1 data [14]. 